\title{
Improving the accuracy of a solid spherical source radius and depth estimation using the diffusion equation in fluorescence reflectance mode
}

\author{
Marjaneh Hejazi ${ }^{11,2}$, Florian Stuker ${ }^{+3}$, Divya Vats 3 and Markus Rudin*3,4
}

\author{
* Correspondence: \\ rudin@biomed.ee.ethz.ch \\ 3 Institute for Biomedical \\ Engineering, University and ETH \\ Zurich, Wolfgang-Pauli-Str. 10, \\ 8093 Zurich, Switzerland \\ + Contributed equally \\ Full list of author information is \\ available at the end of the article
}

\begin{abstract}
Background: Non-invasive planar fluorescence reflectance imaging (FRI) is used for accessing physiological and molecular processes in biological tissue. This method is efficiently used to detect superficial fluorescent inclusions. FRI is based on recording the spatial radiance distribution (SRD) at the surface of a sample. SRD provides information for measuring structural parameters of a fluorescent source (such as radius and depth). The aim of this article is to estimate the depth and radius of the source distribution from SRD, measured at the sample surface. For this reason, a theoretical expression for the SRD at the surface of a turbid sample arising from a spherical light source embedded in the sample, was derived using a steady-state solution of the diffusion equation with an appropriate boundary condition.
\end{abstract}

Methods: The SRD was approximated by solving the diffusion equation in an infinite homogeneous medium with solid spherical sources in cylindrical geometry. Theoretical predications were verified by experiments with fluorescent sources of radius 2-6 mm embedded at depths of 2-4 $\mathrm{mm}$ in a tissue-like phantom.

Results: The experimental data were compared with the theoretical values which shows that the root mean square (RMS) error in depth measurement for nominal depth values $\mathrm{d}=$ $2,2.5,3,3.5,4 \mathrm{~mm}$ amounted to $17 \%, 5 \%, 2 \%, 1 \%$ and $5 \%$ respectively. Therefore, the average error in depth estimation was $\leq 4 \%$ for depths larger than the photon mean free path.

Conclusions: An algorithm is proposed that allows estimation of the location and radius of a spherical source in a homogeneous tissue-like phantom by accounting for anisotropic light scattering effect using FRI modality. Surface SRD measurement enabled accurate estimates of fluorescent depth and radius in FRI modality, and can be used as an element of a more general tomography reconstruction algorithm.

\section{Background}

Optical imaging methods such as fluorescence reflectance imaging (FRI), bioluminescent imaging and fluorescent tomography are used both in the areas of clinical research, such as dermatological and intra-operative imaging and in pre-clinical research for small animal imaging [1]. A conventional FRI system records the spatial distribution of the scattered fluorescent light at the surface of a sample. This light distribution contains sufficient information for determining structural parameters such as radius and depth of fluorescence probe within biological tissue assuming a distribution of spherical sources. Information on depth

(c) 2010 Hejazi et al; licensee BioMed Central Ltd. This is an Open Access article distributed under the terms of the Creative Commons Attribution License (http://creativecommons.org/licenses/by/2.0), which permits unrestricted use, distribution, and reproduction in any medium, provided the original work is properly cited. 
and radius is required for deriving quantitative information on the source intensity, which may be translated into information of molecular and cellular processes within the tissue.

Recently, methods of the depth reconstruction for fluorescence molecular tomography have been widely studied [2-4]. In transmission mode, D'Andrea et al. [5] reported an analytical algorithm to recover the coordinates of a fluorescent inclusion embedded in a turbid medium with accuracy better than $2 \mathrm{~mm}$. Kuo et al.[6] developed a three dimensional reconstruction method for localizing and determining the photon flux of the sources only from a single view. The experimental evaluation revealed that the average error in depth reconstruction for a point source in a sample tissue was $3 \%$ for depth larger than $7 \mathrm{~mm}$ and exceeded $50 \%$ for the superficial depths $(\leq 2.5 \mathrm{~mm})$ [7]. The lack of reconstruction accuracy was attributed to limitations in the assumption that photons were scattered isotropically in biological tissue. Eisdath et al. [8] proposed a theoretical model of photon migration in reflectance mode for localizing the fluorescent inclusion deeply embedded in a turbid media. The source coordinates were obtained with an average error of $4 \%$ at $3 \mathrm{~mm}$ and less than $10 \%$ for depths larger than $6 \mathrm{~mm}$. Up to our knowledge, the radius estimation for superficial fluorescent inclusions in tissue like phantoms has not been reported in FRI modality to date.

The objective of this article is to improve the accuracy of the estimation of source depth and radius in reflection mode by accounting for anisotropic photon scattering. The proposed algorithm can also be regarded as important part of tomographic reconstruction algorithms.

\section{Methods}

\section{Theoretical Background}

The intensity distribution at the surface of a turbid medium is described by a spatial radiance distribution (SRD). This SRD was estimated by solving the diffusion equation in an infinite homogeneous medium with solid spherical sources of radius $R$ with its center located at depth $z$.

For deriving the theoretical SRD, an optical imaging system with the geometry depicted in Fig. 1 was chosen. It consisted of a slab of thickness d characterized by an absorption coefficient $\mu_{a}$ and a reduced scattering coefficient $\mu_{s}^{\prime}$, both with values corresponding to biological tissue.

A spherical light source is placed at the origin of a cylindrical coordinate system $\rho, \varphi z$. The SRD in a highly scattering medium is obtained from solving the diffusion equation for photon propagation $[9,10]$ :

$$
-\nabla(D(\mathbf{r}) \nabla \Phi(\mathbf{r}))+\mu_{a}(\mathbf{r}) \Phi(\mathbf{r})-S(\mathbf{r})=0 \quad \mathbf{r} \in \Omega
$$

where $\Phi(\mathbf{r})$ is the photon fluence (photons $/ \mathrm{cm}^{2}$ ) at location $\mathbf{r}$ with the radius given by $r=\sqrt{\rho^{2}+z^{2}}$ for $z \leq d, \Omega$ describes the region of interest, $S(\mathbf{r})$ an isotropic source term, while the diffusion coefficient $D(\mathbf{r})=3\left(\mu_{a}(\mathbf{r})+\mu_{s}^{\prime}(\mathbf{r})\right)$, accounts for diffusive photon propagation. This equation is derived from the adiative transfer equation [11] by applying the 


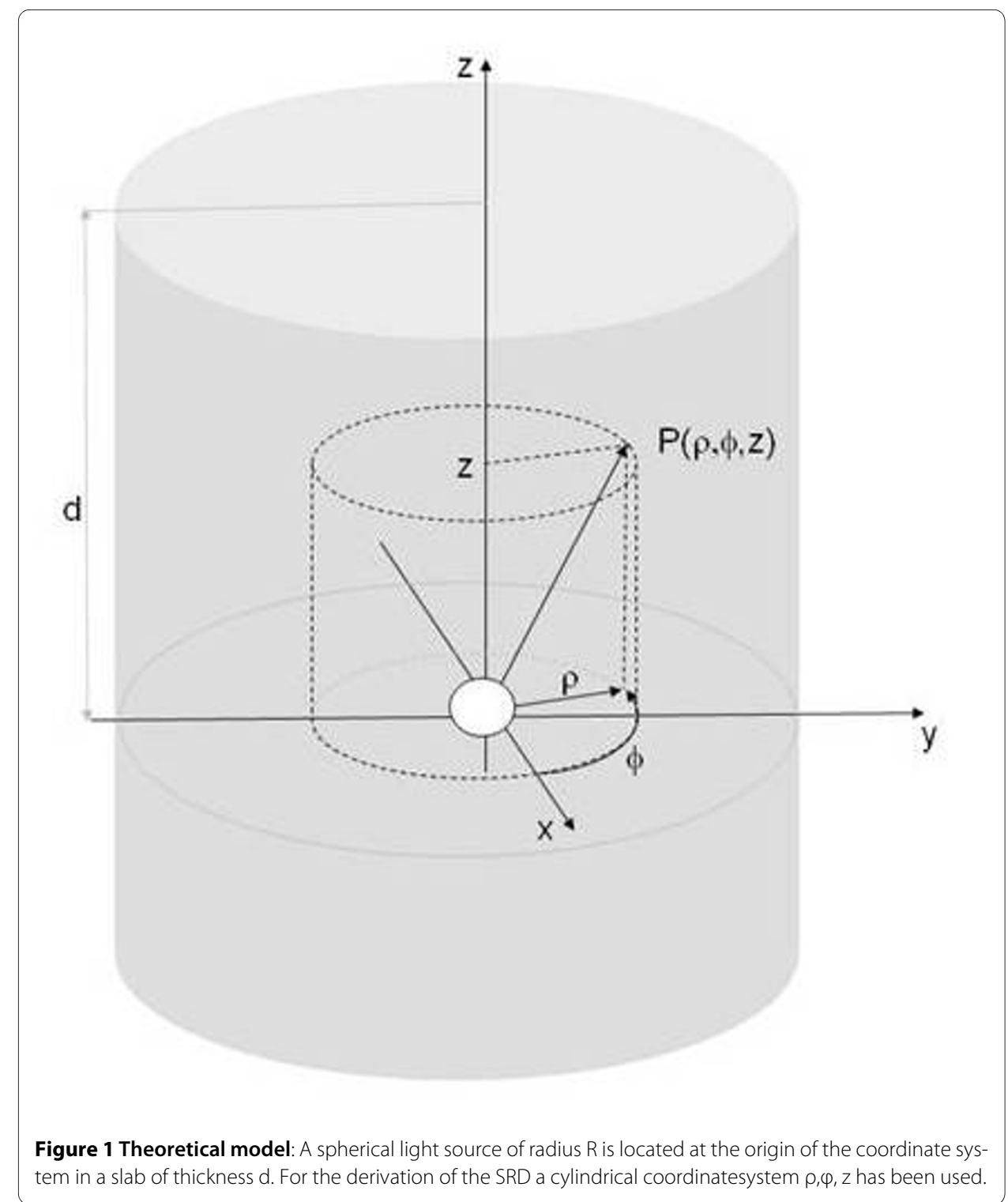

diffusion approximation and is valid for highly scattering media of a thickness greater than the photon mean-free path length.

Recently, the steady-state solution of the diffusion equation with a solid spherical source of radius $R$ embedded in an infinite homogenous medium has been formulated as [12]:

$$
\Phi(\mathbf{r}, \mathrm{R})=\frac{3 P \exp \left(-\mu_{\text {eff }} r\right)}{8 \pi D r R^{3} \mu_{\text {eff }}^{3}} \alpha(R)
$$

with

$$
\alpha(R)=\exp \left(R \mu_{e f f}\right)-\exp \left(-R \mu_{e f f}\right)+R \mu_{e f f}\left(\exp \left(R \mu_{e f f}\right)+\exp \left(-R \mu_{e f f}\right)\right)
$$


where $P$ is the source power $\mu_{\text {eff }}=\left(\mu_{a} / D\right)^{1 / 2}=\sqrt{3 c \mu_{a}\left(\mu_{a}+\mu_{s}^{\prime}\right)}$ denotes the effective attenuation coefficient, while $\alpha(R)$ stands for the exponential terms given in brackets.

$\Phi(\mathbf{r}, R)$ at the interface between two media with different refractive indices can be approximated by an index-mismatched Robin-type boundary condition. This condition for $\Phi(\mathbf{r}, R)$ on $\partial \Omega$ is described in the following formula [13,14]:

$$
\Phi(\mathrm{r}, \mathrm{R})+2 A(\mathbf{r}) \mathrm{D}(\mathbf{r})(\mathbf{n}(\mathbf{r}) \cdot \nabla \Phi(\mathrm{r}, \mathrm{R}))=0 \quad \mathbf{r} \in \partial \Omega
$$

The term $\mathbf{n}$ is the unit vector normal to a surface element $\partial \Omega$ and $A$ is a parameter governing the internal reflection at the boundary. The value of $A=(1+\mathrm{R}) /(1-\mathrm{R})$ depends on the relative refractive index mismatch between tissue and air and can be derived from Fresnel's law, with $\mathrm{R}$ as [14]:

$$
\mathrm{R} \approx-1.4399 n^{-2}+0.7099 n^{-1}+0.6681+0.0636 n
$$

and $n=n_{a} / n_{t}, n_{a}$ and $n_{t}$ being the refractive indices for air and tissue, respectively. This equation yields $A=2.51$ for the air-phantom interface with typical value of $n=1.33$ [15]. Based on the boundary condition the measured fluence rate on $\partial \Omega$ is $[13,16]$.

$$
\Phi(\mathbf{r})=-D(\mathbf{r})(\mathbf{n} \cdot \nabla \Phi(\mathbf{r}))=\Phi(\mathbf{r}) /(2 A(\mathbf{r})) \quad \mathbf{r} \in \partial \Omega
$$

$\Phi(\mathbf{r}, R)$ can be converted to the radiance $L_{o}(\mathrm{r}, \mathrm{R})=\frac{1}{4 \pi} \Phi(\mathrm{r}, \mathrm{R})$ for a perfectly isotropic light distribution:

$$
L_{o}(\mathbf{r}, R)=\frac{0.57 P \exp \left(-\mu_{e f f} r\right)}{32 \pi^{2} D(\mathbf{r}) r R^{3} \mu_{e f f}^{3}} \alpha(R)
$$

Because the radiance in biological tissue is slightly anisotropic and angle dependent due to the large number of scattering events, an angular dependent radiance $L(\mathbf{r}, R, \hat{s})$ is introduced which accounts for anisotropic optical properties of the tissue at $\mathbf{r}$ in direction of $\hat{\mathbf{s}}$, i.e the expression for the radiance (Eq. (6)) is expanded according to [17]:

$$
L(\mathbf{r}, R, \mathbf{s})=L_{o}(\mathbf{r}, R)+\frac{3}{4 \pi} \mathbf{F}(\mathbf{r}) \cdot \mathbf{s},
$$

with the second term denoting the net energy flux $\mathbf{F}(\mathbf{r})=-D(\mathbf{r}) \nabla \Phi(\mathbf{r}, R)$ at a distance $\mathrm{r}$ from the sphere in direction $\hat{\mathbf{s}}[16]$. Combining the various equations one obtains:

$$
L(\mathbf{r}, R, \mathbf{s})=\frac{0.57 \Phi(\mathbf{r}, R)}{32 \pi}\left(\frac{1+3 D(\mathbf{r})\left(r \mu_{e f f}+1\right)}{r^{2}}\right) \mathbf{s} \cdot \mathbf{r}
$$


which for $\mathbf{r} S$ corresponds to the radiance distribution at the surface $S$ of the sample that is generated by a spherical light source of radius $R$, i.e to the SRD.

\section{Experimental Evaluation}

For experimental evaluation, a commercial in-vivo fluorescence imaging system (Maestro, Cri Woburn, USA) has been used with a geometry depicted in Fig. 2. The system consists of a fiber-delivered Xenon excitation source $(\lambda=500-950 \mathrm{~nm}, 30 \mathrm{~W})$, a standard excitation and emission filter set and in addition a liquid crystal tunable filter (LCTF). The LCTF transmits a narrow bandwidth $(10 \mathrm{~nm})$ of the emitted fluorescence light to a 1.3 megapixel CCD camera. A 615-665 nm excitation filter and a $700 \mathrm{~nm}$ long-pass emission filter were used for the evaluation.

The phantom was made from $6 \mathrm{~g}$ agarose (BioGene, Kimbolton, UK), $24 \mathrm{ml}$ Intralipid 20\% (Fresenius SE, Bad Homburg, Germany) and $18 \mu$ Indian ink (Pelikan Holding, Schindellegi, Switzerland) [18] dissolved in $600 \mathrm{ml}$ water. The optical properties of the phantom (with radius $10 \mathrm{~cm}$ ) were measured with a frequency-domain tissue oximeter (oxiplexTS, ISS, Champaign, USA). The reduced scattering and absorption coefficients, at $692 \mathrm{~nm}$, were then found to be $\mu_{s}^{\prime}=8 \mathrm{~cm}^{-1}$ and $\mu_{\mathrm{a}}=0.11 \mathrm{~cm}^{-1}$ respectively. The same

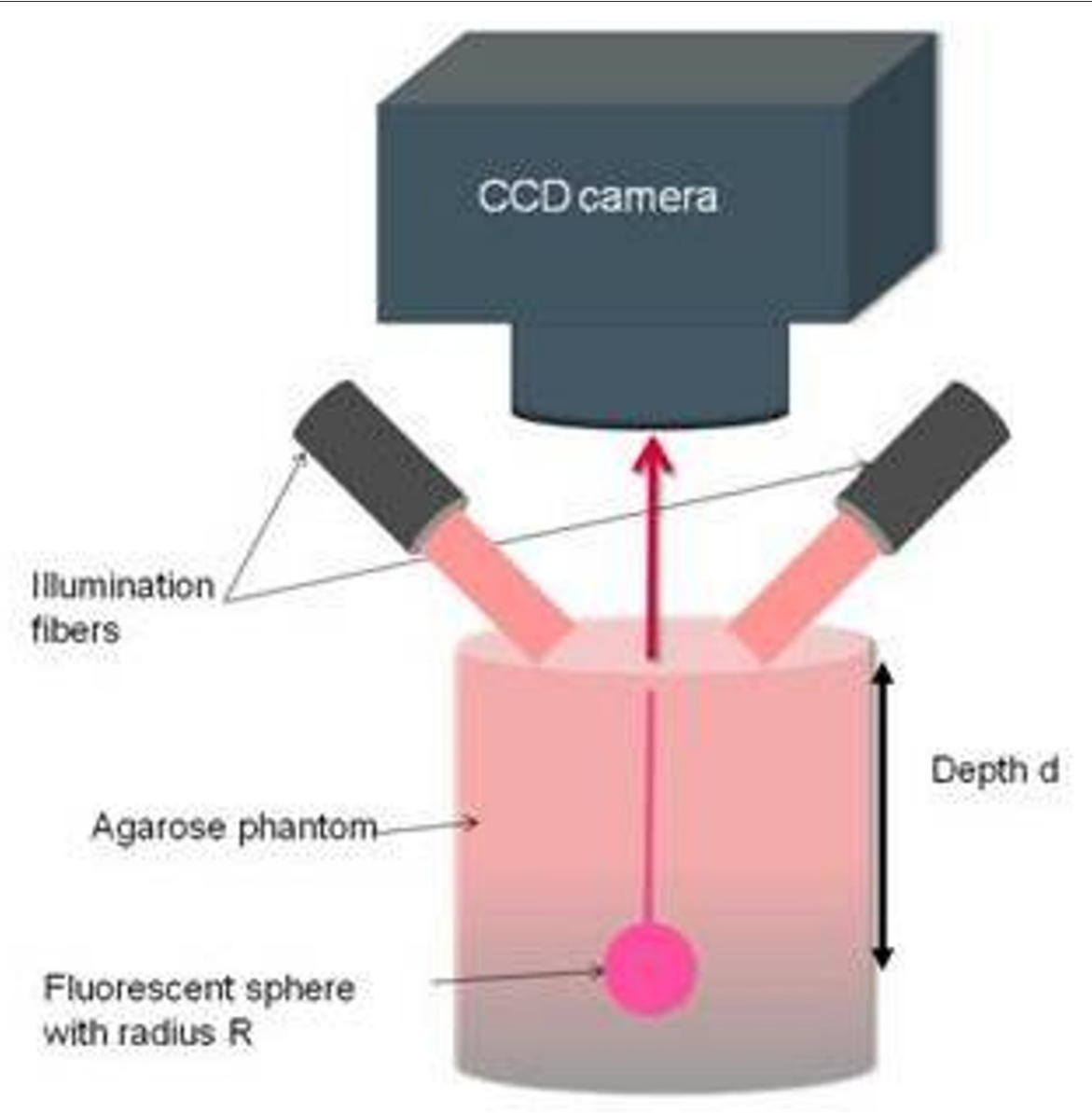

Figure 2 Geometry of experimental setup for determining the SRD using fluorescence imaging in reflection mode. 
mixture was poured into four plexiglass molds of $6 \times 6 \mathrm{~cm}$ and thickness ranging from 2 $\mathrm{mm}$ to $4 \mathrm{~mm}$. The width of the mould was more than 10 penetration depths $(0.6 \mathrm{~cm})$ which fulfilled the requirement for a semi-infinite boundary medium [19]. After solidification, the agarose layers were removed from the molds and positioned by a fixed holder on the top of solid spherical source. The sources with radius 2-6 mm contained $5 \mathrm{mg} / \mathrm{l}$ near-infrared quantum dot (Qdot 705 ITK, Invitrogen, Basel, Switzerland) with emission wavelength at $705 \mathrm{~nm}$.

The optical images were recorded for varying thickness $d$ and denoised by a bandpass filter [20]. The bandpass filter consists of a Gaussian low-pass filter and a boxcar kernel which was used as a high-pass filter. First, a low pass filtered image was generated by convolving the diffuse image with the Gaussian filter, a high-pass filtered image was then obtained by convolving the diffuse image with the boxcar function. The difference between low-pass filtered image and high-pass filtered image allowed extraction of the final image for quantitative analysis.

\section{Results}

Fig. 3a displays the intensity distribution at the sample surface for a spherical quantum dot source of radius $6 \mathrm{~mm}$ located at $\mathrm{d}=3.5 \mathrm{~mm}$. For excitation and detection bandpass filters have been used. The original image was filtered using the bandpass filter (Fig. 3b). The full width at half maximum (FWHM) of intensity distribution (SRD) was derived using Gaussian fitting and amounted to $5.5 \mathrm{~mm}$ (Fig. 3c).

The experimental FWHM data were compared with the theoretical values obtained as FWHM of L(r, R)(Eq. (5)) as function of $d$ and radius (Fig. 4). Source radii range from 2 $\mathrm{mm}$ to $6 \mathrm{~mm}$, while $\mathrm{d}$ was varied between $2 \mathrm{~mm}$ and $4 \mathrm{~mm}$. In general, good agreement between experimental and theoretical FWHM values has been found. Error estimation revealed that the RMS of depth measurement errors for depth values $d=2,2.5,3,3.5,4$ $\mathrm{mm}$ were $17 \%, 5 \%, 2 \%, 1 \%$ and $5 \%$ respectively, for the source radii $2-6 \mathrm{~mm}$ covered in our experiment (Fig. 5).

\section{Discussion and Conclusions}

In this work, we have shown that the structural parameters of a spherical source (depth and radius) can be estimated by measuring the spatial radiance distribution (SRD) at the sample surface. The SRD at the surface of a turbid medium was derived by calculating a steady-state solution of the diffusion equation using the Robin boundary condition.

The accuracy of the theoretical model was validated by fluorescence reflectance imaging of a hemispace homogeneous phantom containing fluorescent source. The comparison showed the RMS of depth measurement errors for depth values $d=2,2.5,3,3.5,4$ mm were $17 \%, 5 \%, 2 \%, 1 \%$ and $5 \%$ respectively. As discussed in previous studies, the relatively large (17\%) overestimation at a depth of $2 \mathrm{~mm}$ was attributed to the inaccuracy of the diffusion approximation for distances comparable to the mean free path $\left(1 /\left(\mu_{s}^{\prime}+\mu_{\mathrm{a}}\right)\right.$ of the photon propagation in a turbid medium [15]. For depth values exceeding $4 \mathrm{~mm}$ 


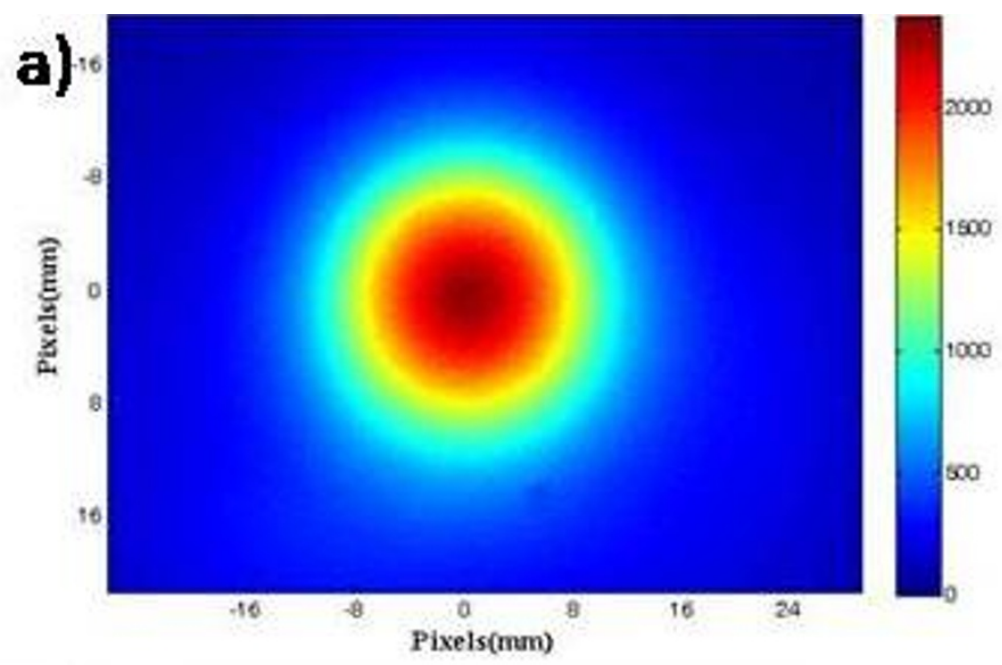

b)
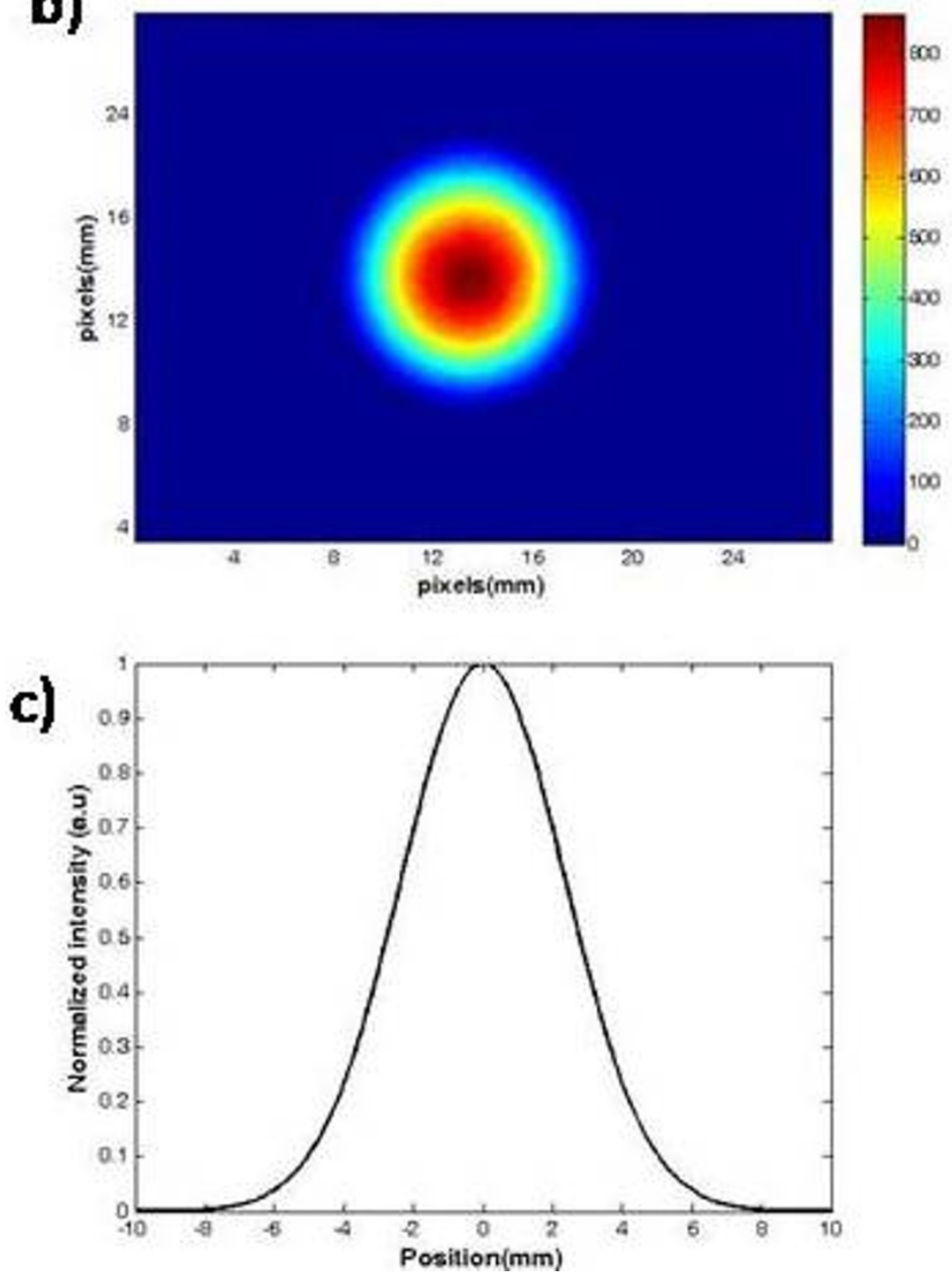

Figure $3 \mathrm{a}$ ) The diffuse image of a QD source of radius $6 \mathrm{~mm}$ in the depth of $3.5 \mathrm{~mm}$ at $620 \mathrm{~nm}$. b) The SRD was extracted by using the bandpass filter. c) The full width at half maximum (FWHM) of the SRD was obtained from the filtered SRD using Gaussian fitting. 


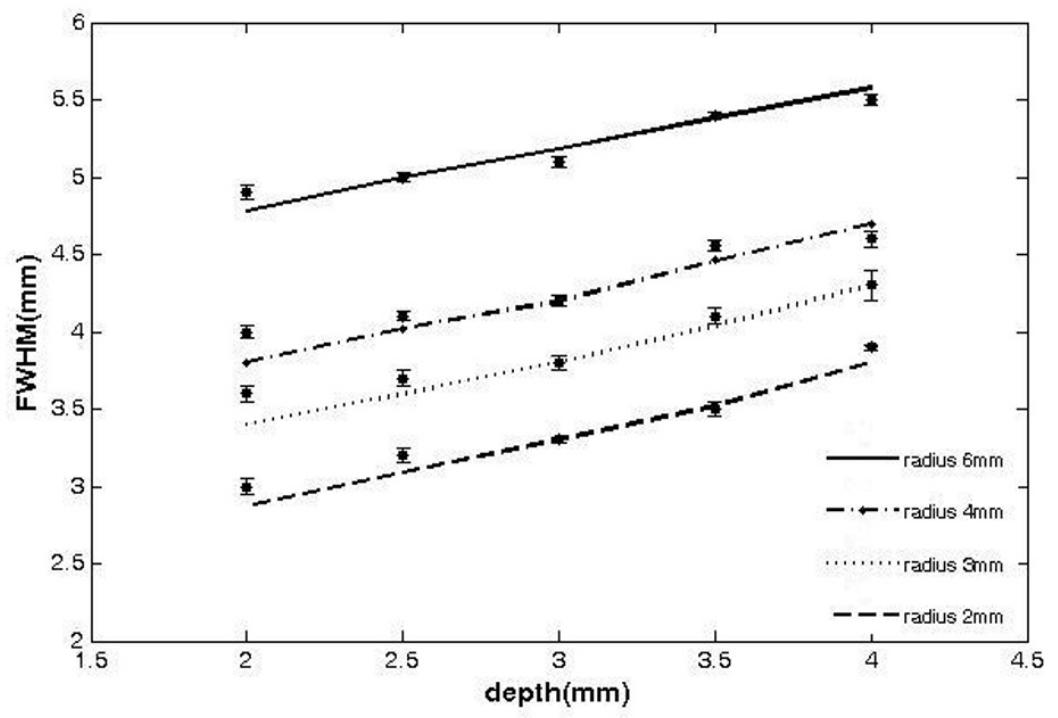

Figure 4 a) FWHM as a function of the depth for the fluorescent sources of radius 2-6 $\mathrm{mm}$ located in a highly turbid media. The experimental and the theoretical data are represented by lines and solid dots respectively. The error bars denote the standard deviation of five measurements.

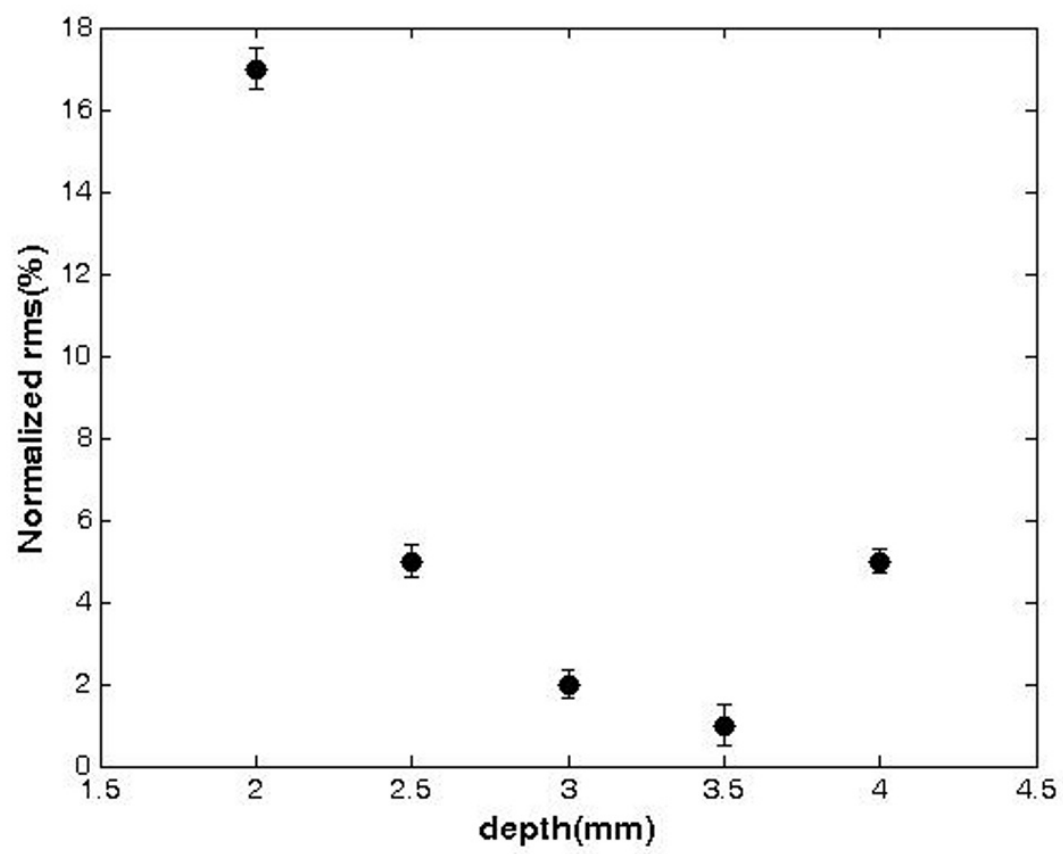

Figure 5 RMS error of the depth reconstruction as function of depth $(\mathrm{mm})$ for source radii $\mathrm{R}=\mathbf{2}$ to 6 $\mathbf{m m}$. The normalized RMS was obtained as $R M S=N^{-1} d_{0}^{-1} \sqrt{\sum_{i=1}^{N}\left(d_{\exp , i}-d_{0}\right)^{2}}$ with $d_{\text {exp }, i}$ and $d_{0}$ being the experimental and nominal depths, respectively, where $N$ is the number of measurements. 
the decrease in fluorescence intensity at the sample surface became a limiting factor; as a consequence of decreased signal-to-noise ratios extraction of accurate SRDs using the denoising method described became difficult and significant errors in FWHM estimation arose. Comparing our method with the procedure described earlier [6] we obtained improved depth estimates close to the sample surface (5\% versus $45 \%$ average error). This might indicate the importance of accounting for the anisotropic distribution of the scattered light in highly turbid media when deriving the SRD, in particular when analyzing source locations within a few millimeter from the sample surface. Eisdath et al. [8] have developed a model for reflectance mode imaging for localizing a fluorescent source in three dimensions. Their results showed that the average error in source localization was less than $10 \%$ for depths larger than $6 \mathrm{~mm}$. However, when using a fluorescence reflectance system SNR becomes a limiting factor for deriving accurate information on source position and dimension within a turbid medium for depth values large than 5 $\mathrm{mm}$.

The algorithm in its current form is not suited for the accurate determination of source location and radius in irregular biological tissues, in particular when considering reconstruction from a single view. The major problem when translating the model to an irregular and heterogeneous sample is that the solution of the inverse problem for the fluorescent source structural parameter does not yield a unique SRD from measurements at a single wavelength $[4,6]$. Next steps in the model development are therefore extension to multiple wavelengths, which will improve the accuracy of the reconstructed source distributions $[4,21]$. A multi-wavelength algorithm can recover the source depth and radius due to the wavelength dependence of SRD (Eqs. (3) and (5)).

In conclusion, an algorithm is proposed that allows estimation of the location and radius of a spherical source in a homogeneous tissue-like phantom by accounting for anisotropic light scattering effect using FRI modality. Experimental validation revealed the accuracy and limitations of the current model. It is obvious that heterogeneity of optical parameters as found in biological tissues will affect the accuracy of the source reconstruction algorithm. These effects will be analyzed in future work.

Competing interests

The authors declare that they have no competing interests.

Authors' contributions

$\mathrm{MH}$ and FS participated in the design of the study, derived the theoretical formula, performed the experimentscarried drafted and revised the manuscript. DV participated in the experimental evaluation and helped to revise the manuscript. MR participated in the theoretical study design, supervised the experimentd and revised the manuscript. All authors read and approved the final manuscript.

Acknowledgements

The authors would like to thank the Swiss National Science Foundation (SNF 310000-112835) for generous funding.

Author Details

1Medical Physics and Biomedical Engineering Department, School of Medicine, Tehran University of Medical Sciences,

1417613151 Tehran, Iran, ${ }^{2}$ Research Center for Science and Technology in Medicine, Tehran University of Medical Sciences, Imam Khomeini Hospital, Keshavarz Blvd, 1417613151 Tehran, Iran, ${ }^{3}$ Institute for Biomedical Engineering, University and ETH Zurich, Wolfgang-Pauli-Str. 10, 8093 Zurich, Switzerland and 4Institute of Pharmacology and

Toxicology, University Zürich, Winterthurerstr. 190, 8057 Zurich, Switzerland 


\section{References}

1. Weissleder R, Ntziachristos V: Shedding light onto live molecular targets. Nat Med 2003, 9:123-128

2. Ntziachristos $V$, Tung C, Bremer C, Weissleder R: Fluorescence molecular tomography resolves protease activity in vivo. Nat Med 2002, 8:757-760.

3. Ntziachristos $V$, Weissleder R: Experimental three-dimensional fluorescence reconstruction of diffuse media by use of a normalized Born approximation. Opt Lett 2001, 26:893-895.

4. Dehghani H, Davis SC, Jiang SD, Pogue BW, Paulsen KD, Patterson MS: Spectrally resolved bioluminescence optical tomography. Opt Lett 2006, 31:365-367.

5. D'Andrea C, Spinelli L, Comelli D, Valentini G, Cubeddu R: Localization and quantification of fluorescent inclusions embedded in a turbid medium. Phys Med Biol 2005, 50:2313-2327.

6. Kuo C, Coquoz O, Troy TL, Xu H, Rice BW: Three-dimensional reconstruction of in vivo bioluminescent sources based on multispectral imaging. J Biomed Opt 2007, 12:1-12. 024007

7. Virostko J, Powers AC, Jansen DE: Validation of luminescent source reconstruction using single-view spectrally resolved bioluminescence images. Appl Opt 2007, 46:2540-2547.

8. Eidsath A, Chernomordik V, Gandjbakhche A, Smith P, Russo A: Three-dimensional localization of fluorescent masses deeply embedded in tissue. Phys Med Biol 2002, 47:4079-4092.

9. Schultz R, Ripoll J, Ntziachristos V: Experimental fluorescence tomography of tissues with noncontact measurements. IEEE Trans Med Imag 2004, 23:492-500.

10. Arridge SR, Schweiger M, Hiraoka M, Delpy DT: A finite element approach for modeling photon transport in tissue. Med Phys 1993, 20:299-309.

11. Wang LV: Biomedical optics: Principles and imaging. John Wiley and Sons; 2007.

12. Shen $\mathrm{H}$, Cong W, Qian X, Durairaj K, Wang G: Numerical study on the validity of the diffusion approximation for computational optical biopsy. J Opt Soc Am A 2007, 24:423-429.

13. Cong WX, Wang G, Kumar D, Liu Y, Jiang M, Wang LH, Hoffman EA, McLennan G, McCray PB, Zabner J, Cong A: A practical reconstruction method for bioluminescence tomography. Opt Express 2005, 13:6756-6771.

14. Schweiger M, Arridge SR, Hiraoka M, Delpy DT: The finite element method for the propagation of light in scattering media: Boundary and source conditions. Med Phys 1995, 22:1779-1792.

15. Hawrysz J, Roy R, Sevick-Muraca EM: Influence of the refractive index-mismatch at the boundaries measured in fluorescenceenhanced frequency-domain photon migration imaging Anuradha Godavarty. Opt Express 2002, 10:653-662.

16. Comsa DC, Farrell TJ, Patterson MS: Quantification of bioluminescence images of point source objects using diffusion theory models. Phys Med Biol 2006, 51:3733-3746

17. Welch AJ, Gemert MJCV: Optical thermal response of laser-irradiated tissue. Plenum Press, New York; 1995.

18. Cubeddu R, Pifferi A, Taroni P, Torricelli A, Valentini G: A solid tissue phantom for photon migration studies. Phys Med Biol 1997, 42:1971-1979.

19. Lin SP, Wang L, Jacques SL, Tittel FK: Measurement of tissue optical properties by the use of oblique-incidence optical fiber reflectometry. Appl Opt 1997, 36:136-143.

20. Crocker JC, Grier DG: :Methods of digital video microscopy for colloidal studies. J Colloid Interface Sci 1996, 179:298-310.

21. Wang G, Li Y, Jiang M: Uniqueness theorems in bioluminescence tomography. Med Phys 2004, 31:2289-299.

doi: $10.1186 / 1475-925 X-9-28$

Cite this article as: Hejazi et al., Improving the accuracy of a solid spherical source radius and depth estimation using the diffusion equation in fluorescence reflectance mode BioMedical Engineering OnLine 2010, 9:28

\section{Submit your next manuscript to BioMed Central and take full advantage of:}

- Convenient online submission

- Thorough peer review

- No space constraints or color figure charges

- Immediate publication on acceptance

- Inclusion in PubMed, CAS, Scopus and Google Scholar

- Research which is freely available for redistribution 